\title{
On the numerical resolution of the bottom layer in simulations of oceanic gravity currents
}

\author{
N. Laanaia, A. Wirth, J. M. Molines, B. Barnier, and J. Verron \\ LEGI/MEOM/CNRS, Laboratoire des Ecoulements Géophysiques et Industriels, UMR 5519, BP 53, \\ 38041 Grenoble cedex 9, France \\ Received: 11 February 2010 - Published in Ocean Sci. Discuss.: 4 March 2010 \\ Revised: 25 May 2010 - Accepted: 26 May 2010 - Published: 8 June 2010
}

\begin{abstract}
The role of an increased numerical vertical resolution, leading to an explicit resolution of the bottom Ekman layer dynamics, is investigated. Using the hydrostatic ocean model NEMO-OPA9, we demonstrate that the dynamics of an idealised gravity current (on an inclined plane), is well captured when a few (around five) sigma-coordinate levels are added near the ocean floor. Such resolution allows to considerably improve the representation of the descent and transport of the gravity current and the Ekman dynamics near the ocean floor, including the important effect of Ekman veering, which is usually neglected in today's simulations of the ocean dynamics.

Results from high resolution simulations (with $\sigma$ and zcoordinates) are compared to simulations with a vertical resolution commonly employed in today's ocean models. The latter show a downslope transport that is reduced by almost an order of magnitude and the decrease in the along slope transport is reduced six-fold. We strongly advocate for an increase of the numerical resolution at the ocean floor, similar to the way it is done at the ocean surface and at the lower boundary in atmospheric models.
\end{abstract}

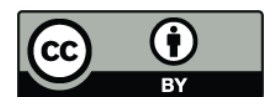

Correspondence to: A. Wirth (achim.wirth@hmg.inpg.fr)

\section{Introduction}

The realism of numerical models of the ocean dynamics depends on their capability to correctly represent the important processes, at large and also at small scales. The dynamics of gravity currents was identified as a key process governing the strength of the thermohaline circulation and its heat transport from low to high latitudes (Willebrand et al., 2001).

Oceanic gravity currents are small compared to the basin scales in the horizontal and vertical directions, only about $100 \mathrm{~km}$ wide and a few hundred metres thick, but they have a substantial impact on the global climate dynamics.

A conspicuous feature of todays numerical simulations of the ocean circulation is their increased vertical resolution at the ocean surface. It is the physics of the near surface processes and their importance for the large scale ocean circulation that imposes the grid refinement near the surface. More precisely, the ocean is forced predominantly by fluxes of inertia and heat at its surface. These fluxes give rise to the so called planetary boundary layer dynamics (PBL). It is through this boundary layer, that the surface forcing propagates to the interior ocean. The quality of a simulation of the ocean dynamics is thus governed by the representation of the PBL dynamics. The important dynamical processes in the surface PBL have a smaller vertical scale than the dynamics in the interior ocean and this fact has to be represented in the

Published by Copernicus Publications on behalf of the European Geosciences Union. 
grid of the numerical model, leading to the above mentioned refinement near the surface.

At the ocean floor a similar PBL develops. This feature is, however, rarely reflected in the structure of the vertical resolution. To the contrary, the grid size is usually an increasing function of depth, leading to the sparsest resolution at the ocean floor. The PBL dynamics at the ocean floor can not be explicitly represented with such a vertical grid. Please note, that in numerical models of the dynamics of the atmosphere, a grid refinement near the earth's surface is commonly employed to resolve explicitly a large part of the important processes at this boundary. The dynamics at the ocean floor is however similarly important and involved, as the dynamics at the ocean surface and at the lower boundary of the atmosphere. A large part of the kinetic energy is supposed to be dissipated at the ocean floor representing a major sink of kinetic energy and an important player in the global energy cycle of the ocean dynamics.

Furthermore, a misrepresentation of the PBL dynamics is worse in the bottom layer than in the surface layer, when the momentum balance is considered. Indeed, at the surface the wind shear is imposed and thus also the corresponding Ekman transport, which means, that even with a bad representation of the PBL dynamics the overall Ekman transport is correct in magnitude and direction. In the bottom layer, to the contrary, the shear is a function of the velocity field near the bottom. Getting the velocity field wrong also means, that the Ekman transport is wrong in magnitude and direction. It is through the divergence of the Ekman layer transport that the momentum fluxes at the boundaries are communicated to the ocean interior (see e.g. Pedlosky 1998). The need is especially important when the dynamics of gravity currents are considered. It was demonstrated by Willebrand et al. (2001) that the thermohaline circulation of the North Atlantic in numerical models is strongly influenced by the local representation of the gravity current dynamics in the Denmark Strait.

Many efforts have been made during the last thirty years to parametrise the effect of the bottom PBL on the large-scale ocean dynamics. Parametrisations of varying complexity have been developed to represent various features of the dynamics of gravity currents (see e.g. Killworth and Edwards, 1999; Xu et al., 2006; Legg et al., 2006, 2008). Today there is no generally accepted parametrisation and the representation of gravity currents is considered a major flaw in today's state-of-the-art ocean models. Developing a parametrisation of the Ekman layer dynamics, based on linear homogeneous stationary Ekman layer theory might be of limited use in cases where the Ekman layer is neither linear nor homogeneous nor stationary. Indeed, inertial oscillations and a rapid evolution of the gravity current, make the validity of such parametrisations questionable.

In the present work we explore another direction. Rather than parametrising the total PBL dynamics on the bottom we resolve some of it explicitly, by increasing the resolution near the bottom in the very same way as it is commonly done near the surface. This refinement can easily be implemented in a $\sigma$-coordinate grid, available in several numerical models of the ocean circulation. This idea has, so far to the best of our knowledge, not been explored in detail. The importance of the vertical resolution in gravity current dynamics has already been emphasised by various authors. A fine grid or a grid refinement near the ocean floor was already employed, for example, in Ezer and Weatherly (1990), Winton et al. (1998), Jungclaus (1999), Ezer and Mellor (2004); Ezer (2005). But we like to mention that in these publications, although having a high vertical resolution, the Ekman layer dynamics in the PBL is usually not sufficiently resolved and the effect of vertical resolution on the gravity current dynamics has not been explored in detail. Most recently Legg et al. (2008), varying the vertical viscosity over more than three orders of magnitude rather than the grid resolution, noted that resolving the Ekman layer has a dominant role in determining the descent of the gravity current and favours its downslope movement. Their paper also gives a very nice introduction to, and a review of, recent results using numerical simulations of gravity current dynamics.

In this work we suggest a vertical resolution in ocean models that represents a compromise between calculation time and representation of the important processes. We focus on the importance of vertical resolution in the representation of the dynamics of gravity currents as: (i) gravity currents are affected by the PBL dynamics on the ocean floor, (ii) gravity currents are important features of the large scale circulation, (iii) they represent a difficult problem to simulate numerically and (iv) they have been thoroughly studied in observations, laboratory experiments, numerical models and analytical calculations and the results are discussed in a large number of publications.

The present work is dedicated to the importance of Ekman layer dynamics and its vertical resolution in numerical simulations of gravity currents. This vertical resolution is shown to be of paramount importance for the process considered here. The important problem of errors in the horizontal pressure gradient in $\sigma$-coordinate models, a subject discussed in a large number of publications, is not considered here.

\section{Dynamics and representation of the oceanic bottom boundary layer}

The dynamics in the PBL at the ocean floor is turbulent. The key parameter of its dynamics is the friction velocity:

$u^{*}=\sqrt{\frac{\tau}{\rho}}$,

where $\tau$ is the friction force per unit area exerted by the ocean floor and $\rho$ is the density of the sea water.

The PBL in the ocean can be roughly decomposed into four layers. The first, counting upward from the bottom, is the viscous sub-layer which is only a few millimetres thick 
$\delta_{v} \approx 5 v / u^{*}$ and which is governed by laminar viscous dynamics. Above this layer the dynamics transits in the buffer layer towards the log-layer, which is a few metres thick and governed by turbulent transfer of inertia. The thickness of the buffer-layer is a function of the roughness of the ocean floor. The transfer of inertia is supposed constant throughout the log-layer and its magnitude also depends on the roughness of the ocean floor. In the fourth layer, at even further distance $\delta_{f} \approx 0.2 u^{*} / f$, of a few tens of metres above ground, rotation influences the dynamics and a turbulent Ekman layer develops (see Coleman et al., 1990; McWilliams, 2009).

The dynamics in the first three layers can not be explicitly resolved in ocean models even in a far future. The viscous sub-layer is only a few millimetres thick and the buffer- and log-layer are governed by turbulent structures of only a few metres in size in the vertical and horizontal directions. An explicit resolution asks for grid cells of less than one metre in all three spatial directions and a time step smaller than one second, requirements which are far from being feasible for basin-scale ocean models, with today's and tomorrow's computer resources. The characteristic time scale of the dynamics in the first three boundary layers is faster than the inverse of the Coriolis parameter, which is a prerequisite for an efficient parametrisation in ocean models. The dynamics in the first three bottom layers, viscous-, buffer- and loglayer, is usually parametrised by implementing either: (i) a no slip boundary condition together with an increased vertical turbulent viscosity or (ii) a drag force at the lowest grid cell, which depends linearly or quadratically on the velocity at the first grid point from the wall. The actual drag force per unit area in today's ocean models is often given by $\mathbf{F}=\left(c_{\mathrm{D}} / \rho\right)(|\mathbf{u}|+c) \mathbf{u}$, where $\mathbf{u}$ is the fluid velocity near the ocean floor, $c_{\mathrm{D}}$ the drag coefficient depending on the roughness of the ocean floor and $c$ is a velocity representing processes not explicitly included, as for example the tidal dynamics. This leads to a linear friction force for $u \ll c$ and a quadratic friction force for $u \gg c$. The problem of parametrising the influence of these three layers lies in the determination of the corresponding friction parameters (see Wirth and Verron, 2009; Wirth, 2010).

We prefer to resolve explicitly a part of the dynamics in the Ekman layer rather than to totally parametrise its influence on the dynamics above. The parametrisation of the Ekman layer dynamics is more subtle due to the veering (turning) of the velocity vector in the Ekman layer. When this effect is omitted, as it is currently in most ocean general circulation models, the friction force is not only wrong in magnitude but also in direction. The focus of the present work is on the numerical resolution of the Ekman layer dynamics at the ocean floor.

\section{Idealised oceanic gravity current on the f-Plane}

\subsection{The physical problem considered}

In the experiments presented here we use an idealised geometry, considering an infinite gravity current on an inclined plane with constant slope of $1^{\circ}$ in a rotating frame with a constant Coriolis parameter $f=1.0313 \times 10^{-4} \mathrm{~s}^{-1}$. The water in the gravity current is $\Delta T=0.2 \mathrm{~K}$ colder than the surrounding water, gravity is $g=9.8065 \mathrm{~ms}^{-2}$ and the thermal expansion coefficient equals $2.0^{-4} \mathrm{~K}^{-1}$. This leads to a reduced gravity $g^{\prime}=3.9226 \times 10^{-4} \mathrm{~ms}^{-2}$. In the initial condition the thickness $h(y)$ has a parabolic shape that is $20 \mathrm{~km}$ wide and has a maximum value of $200 \mathrm{~m}$. Initially the velocity is in a geostrophic equilibrium.

When dissipative effects and instability of the gravity current are ignored the gravity current is stationary and travels in the along-slope direction with an average geostrophic velocity of $u=\left(g^{\prime} / f\right) \tan 1^{\circ}$. It is the friction, transmitted by the PBL dynamics, that is responsible for the evolution of the gravity current. Please note that in the rotating case it is friction that makes the gravity current move down-slope, whereas in the non-rotating gravity current friction opposes the down slope movement. This dynamics has been studied numerically in Wirth (2009) using the non-hydrostatic model HAROMOD introduced in Wirth (2004). We are here concerned with the representation of the dynamics in hydrostatic ocean models.

\subsection{The mathematical model}

In the present research we consider two configurations to study the physical problem described above. The first configuration is 2.5 dimensional and the second 3 dimensional. In the $2.5 \mathrm{D}$ configuration we only consider the dynamics within a 2 dimensional vertical slice perpendicular to the geostrophic velocity. We do not allow for variations in the long-stream direction. Although the geometry is 2-D all three components of the velocity vector are retained. The 2.5-D configuration has two advantages. First, it suppresses large scale instability of the gravity current and the formation of large scale eddies, which is beneficial to our goal of looking at the influence of small scale processes in the PBL. Second, a detailed study of the influence of resolution is numerically more feasible in the less costly $2.5-\mathrm{D}$ configuration. The 3-D configuration is then used to verify how the results found in the 2.5-D configuration transfer, qualitatively and quantitatively, to the full 3-D case. That is, we check if the presence of 3-D instabilities and other structures alter the results found in the $2.5-\mathrm{D}$ case.

The domain spans $50 \mathrm{~km}$ in the y-direction (upslope), its maximal depth is $1250 \mathrm{~m}$. The 3-D configuration spans $200 \mathrm{~km}$ in the $\mathrm{x}$-direction which is ten times the initial width of the vein (see below). 
Table 1. List of the 2.5-D exps. The domain spans $50 \mathrm{~km}$ in the $\mathrm{y}$ direction. The number of levels in the vertical zones $\mathrm{Z} 1, \mathrm{Z} 2$ and $\mathrm{Z3}$ (as explained in the text) are given.

\begin{tabular}{llcrrrl}
\hline Exp. & Resolution (ny,nz) & Coord. type & Z1 & Z2 & Z3 & Rem. \\
\hline G01 & $(350.500)$ & z & 98 & 278 & 124 & reference \\
G02 & $(350.200)$ & $\sigma$ & 16 & 64 & 120 & reference \\
G03 & $(350.10)$ & $\sigma$ & 3 & 4 & 2 & \\
G04 & $(16.10)$ & $\sigma$ & 3 & 4 & 2 & \\
G05 & $(350.8)$ & $\sigma$ & 1 & 4 & 2 & \\
G06 & $(350.7)$ & $\sigma$ & 3 & 1 & 2 & \\
G07 & $(350.5)$ & $\sigma$ & 1 & 1 & 2 & \\
G08 & $(350.4)$ & $\sigma$ & 1 & 1 & 1 & \\
G09 & $(250.250)$ & z & 49 & 139 & 62 & convect. adj. \\
\hline
\end{tabular}

The mathematical model used to study the physical configuration introduced above comprises the hydrostatic (primitive) equations subject to a no slip boundary condition at the bottom and an implicit free surface at the top. The vertical eddy viscosity is $4 \times 10^{-3} \mathrm{~m}^{2} \mathrm{~s}^{-1}$ leading to a thickness of the laminar Ekman layer of $\delta_{\mathrm{Ek}}=\sqrt{2 v / f}=8.8 \mathrm{~m}$ and the vertical diffusivity is $1 \times 10^{-4} \mathrm{~m}^{2} \mathrm{~s}^{-1}$. The horizontal viscosity is $v_{\mathrm{h}}=10 \mathrm{~m}^{2} \mathrm{~s}^{-1}$ and the horizontal diffusivity is $\kappa_{\mathrm{h}}=1 \mathrm{~m}^{2} \mathrm{~s}^{-1}$.

\subsection{The numerical model and its vertical resolution}

The above introduced mathematical models are numerically integrated with the numerical model NEMO-OPA9 (see Madec, 2008). The horizontal resolution $(\Delta y=143 \mathrm{~m})$ in the direction of the slope is, with one exception $(\Delta y=3125 \mathrm{~m}$ in G04), the same in all experiments (please see Tables 1 and 2 for details). The horizontal resolution of experiment G04 is typical for high resolution regional models. In the three dimensional experiments $\Delta x=400 \mathrm{~m}$.

The purpose of our research is to evaluate the effect of a vertical grid refinement near the ocean floor and determine the grid structure that is an optimal compromise between accuracy and the cost of calculation. Two types of grid geometries are employed here, both are standard options in the NEMO-OPA9 model. One is the conventional z-coordinate, where all grid-points of a certain level lie at the same depth. The grid structure has a regular orthogonal form. Usually the vertical resolution is a function of depth, with a refinement near the surface and a sparse grid at the bottom. For the case considered here, a high resolution at the topography, the refinement has to extend over the total depth, leading to a uniform grid structure with many grid points in areas where they are not necessarily needed.

The second type of grid geometry is the $\sigma$-coordinate, which is terrain following. The standard Jacobian formulation is used for the calculation of the horizontal pressure gradient (see Madec, 2008, 90 p., of the reference manual). In a $\sigma$-coordinate model the number of levels is equal everywhere so that no grid point is wasted in the vertical. In a $\sigma$-coordinate model grid points of the same level are situated at the same depth relative to the total depth at each horizontal location. This type of grid structure allows for an efficient refinement of the grid near the topography. Except for two experiments all our experiments are performed with a $\sigma$-coordinate system. No 3-D reference experiments (high resolution) experiments have been performed due to the inhibitive computer requirements. The convective adjustment on the tracer (only) is used in all simulations (by increasing the vertical diffusivity to $10 \mathrm{~m}^{2} \mathrm{~s}^{-1}$ ).

We want to emphasise here that the mathematical model presented in the previous subsection has a well defined solution which is, of course, independent of the numerical model employed to approach it. As the numerical models with both types of grids are consistent their solutions will both converge to the mathematical solution when the grid resolution and the corresponding time step are reduced. The question is, however, which of the grids has a faster convergence when numerical costs are equal. The case considered here is clearly in favour of the $\sigma$-coordinate model.

In our numerical simulations we distinguish three zones in the vertical direction (see Fig. 3). The first zone, called $\mathrm{Z} 1$, includes the sea-floor PBL and is about $40 \mathrm{~m}$ thick. The second, called Z2, extends from about 40 to $200 \mathrm{~m}$ from the ocean floor and includes thus the upper part of the vein. The third zone, called Z3, extends from about $200 \mathrm{~m}$ above the ocean floor to the surface and contains no gravity current water. It, nevertheless, has an influence on the dynamics of the gravity current. The vertical resolution is varied between experiments in the three zones to explore its effect on the representation of the gravity current dynamics.

\section{Experiments}

We performed two sets of experiments, the first are 2.5-D and the second 3-D. Both sets are explained in the following two subsections, the results of the experiments are given in the next section. The initial conditions, identical for both sets of experiments, can be seen in Fig. 1, the temperature is constant within the gravity current which has a parabolic shape and the long-slope velocity is geostrophically adjusted, please note that it reverses within the gravity current.

\subsection{Experiments 2.5-D}

We started by performing two reference experiments of the 2.5-D configuration at very high vertical resolution with 500 levels for the $\mathrm{z}$-coordinate and 200 levels for the $\sigma$ coordinate calculations. Please see Table 1 for a concise presentation of all the 2.5-D experiments performed. The first reference experiment (G01), one of two experiments using a $\mathrm{z}$-coordinate, and the second (G02) with a $\sigma$-coordinate, are performed to establish results with which the other experiments can be compared. In experiment G01 the vertical resolution is $2.5 \mathrm{~m}$ everywhere, whereas in experiment G02 

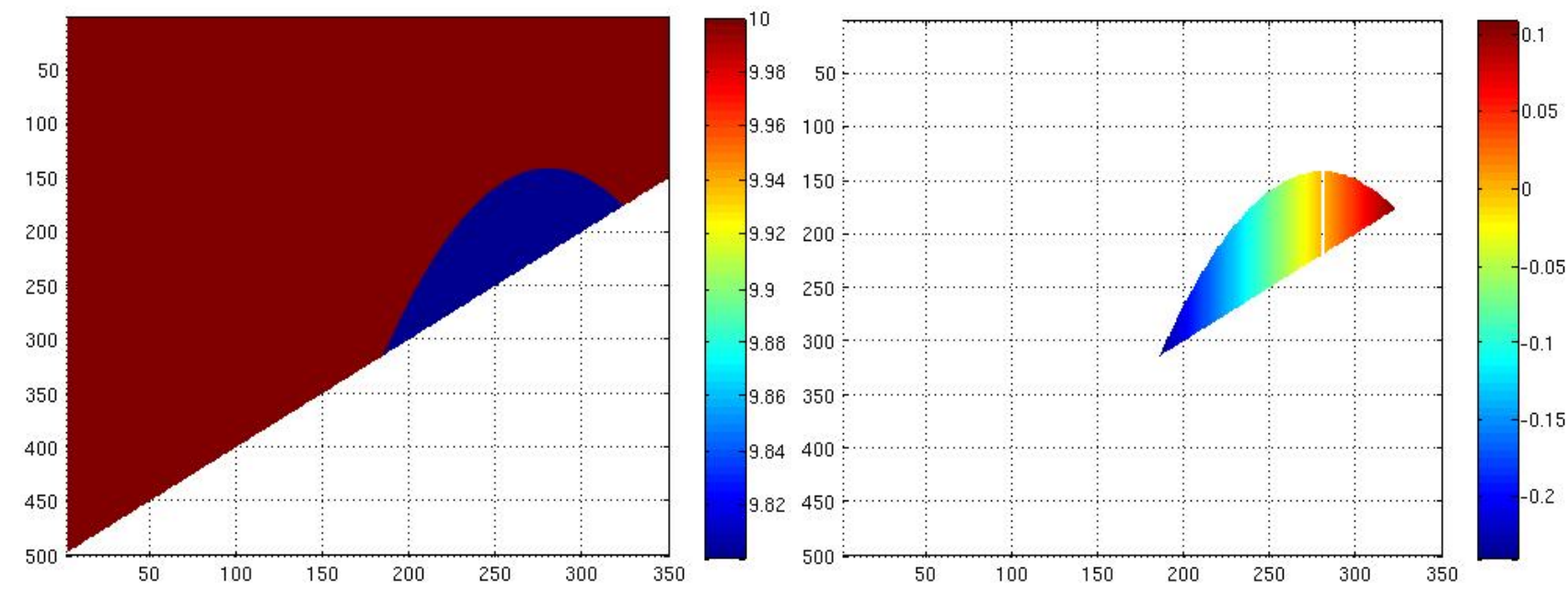

Fig. 1. Initial condition: temperature (left) and geostrophically adjusted velocity $u$ (cross stream component; y-direction is upslope).

the first level is at only $0.3 \mathrm{~m}$ from the ocean floor (measured at the upslope side of the domain). Experiment G02 is thus of higher quality than G01 due to the grid refinement near the ocean floor. In other experiments the resolution is varied in the vertical zones defined above (Z1, Z2 and Z3) to evaluate the lack of resolution in the different zones, each one being representative of different physical processes. For the grids with three levels in the zone $\mathrm{Z} 1$ the first three horizontalvelocity grid points are at $2.5 \mathrm{~m}, 10 \mathrm{~m}$ and $22.5 \mathrm{~m}$ from the ocean floor resolution (on the up slope side of the domain). A typical $\sigma$-coordinate grid with three levels in the zone $\mathrm{Z} 1$ is given in Fig. 3

Experiment G04 has a coarser horizontal with $\Delta y=3.125 \mathrm{~km}$ a value typical for today's state-of-theart regional GCM calculations. A second experiment, G09, with a $z$-coordinate system is performed. It has a coarser resolution (in $\mathrm{x}$ and $\mathrm{z}$ ) as compared to G01 and the convective adjustment is also used on the momentum (by increasing the vertical viscosity to $10 \mathrm{~m}^{2} \mathrm{~s}^{-1}$ ). Such scheme is usually employed in ocean global circulation models (OGCM) integrations to parametrise convective processes at the ocean surface. The convective adjustment procedure used, increases the vertical viscosity and diffusivity many orders of magnitude, to $10 \mathrm{~m}^{2} \mathrm{~s}^{-1}$, when a static instability is detected. This mimics convection and is beneficial near the surface, but destroys the gravity current dynamics as we will demonstrate in the next section.

\subsection{Experiments 3-D}

In the 3-D experiments a reference experiment, as performed for the 2.5-D case, is prohibited by the size of the calculation. We therefore used the resolution in Z1 and Z2, which was found to give good results in the 2.5-D case, experiment G03 (see Table 1 and Sect. 5) as the resolution of our 3-D reference experiment G11 (see Table 2). The higher resolution
Table 2. List of the 3-D exps. (see Table 1 for details).

\begin{tabular}{cccccr}
\hline Exp. & $\begin{array}{c}\text { Resolution } \\
(\text { nx,ny,nz) }\end{array}$ & Coord. type & Z1 & Z2 & Z3 \\
\hline G11 & $(500.350 .12)$ & $\sigma$ & 3 & 4 & 4 \\
G12 & $(500.350 .10)$ & $\sigma$ & 1 & 4 & 4 \\
G13 & $(500.350 .14)$ & $\sigma$ & 0 & 3 & 10 \\
\hline
\end{tabular}

in the zone $\mathrm{Z} 3$ as compared to the 2.5-D experiments is required for the numerical stability of the calculation. Indeed, the eddy dynamics due to large scale instability of the flow, which are suppressed in the 2.5-D calculations, ask for a higher resolution in Z3 (see Sect. 5). The resolution of G11 is shown in Fig. 3.

The effect of a non resolved Ekman layer was studied in experiment G12, its vertical resolution is similar to G05. Experiment G13 at an even sparser resolution is close to what is used in classical high vertical-resolution calculations of gravity current dynamics and OGCM calculations.

\section{Results}

\subsection{Results 2.5-D}

The two reference simulations (G01 and G02) produce very similar results for the temperature structure, compared in Fig. 2 and the velocity field, showing the consistency of the numerical scheme on both numerical grids. The shapes compare very well to the results of non-hydrostatic calculations of Wirth (2009) and to laboratory experiments made on the Coriolis platform in Grenoble (Wirth and Sommeria, 2007), validating the use of a hydrostatic model to numerically simulate gravity current dynamics. The conspicuous feature, 

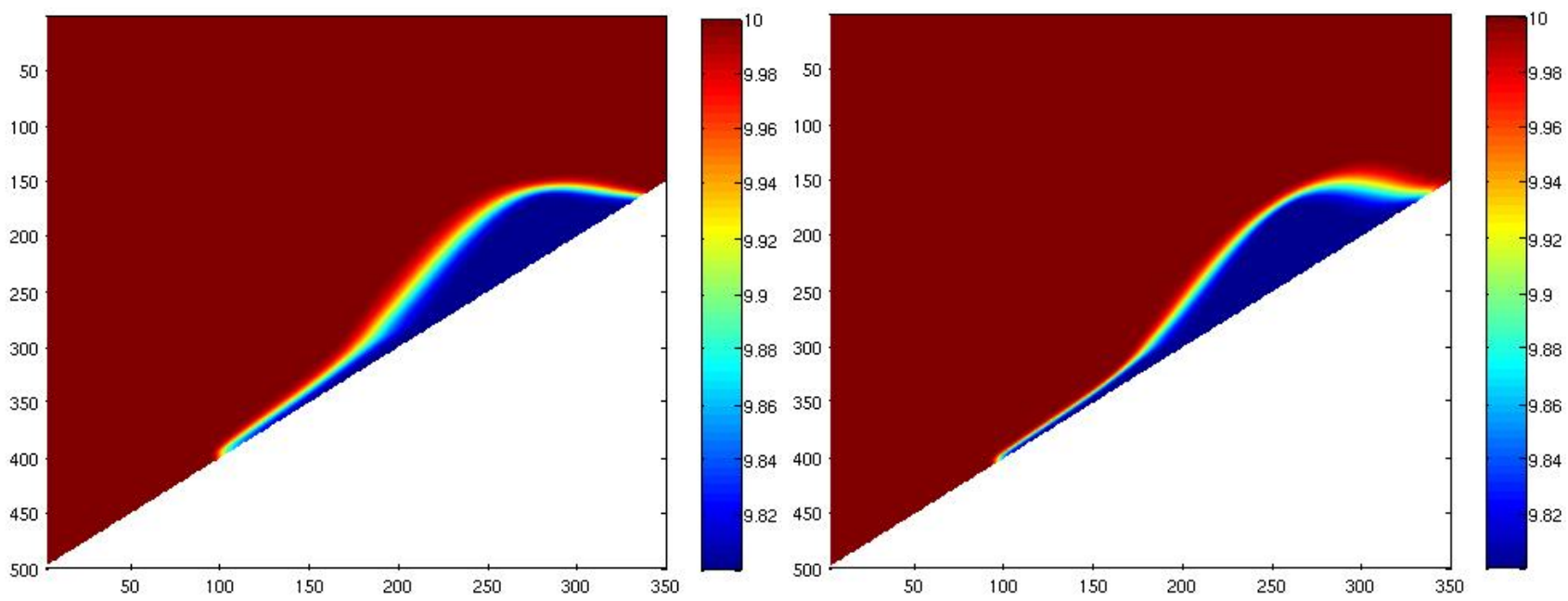

Fig. 2. Temperature structure $\left(\right.$ in $\mathrm{C}^{\mathrm{o}}$ ) in the reference simulations after 7 days; left: $\mathrm{z}$-coordinate (G01) and right: $\sigma$-coordinate $(\mathrm{G} 02)$. Coordinates give grid in the $\mathrm{x}$-direction and the $\mathrm{z}$-direction for the $\mathrm{z}$-coordinate grid. For the $\sigma$-coordinate results are interpolated to the $\mathrm{z}$-coordinate grid (in the z-direction).

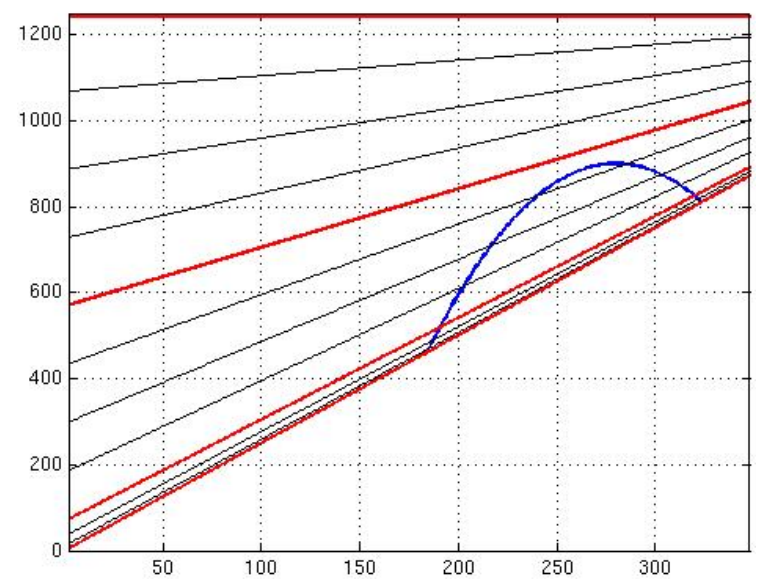

Fig. 3. $\sigma$-coordinates of G11 shown (black lines). Red-lines mark the boundaries of the zones $\mathrm{Z} 1, \mathrm{Z} 2$ and $\mathrm{Z3}$ (bottom to top). Blue line gives initial profile of gravity current.

common in all the laboratory experiments and high resolution numerical simulations, is a vein, the thick part of the gravity current and a thin "friction layer" at its down slope side. The vein descends only slowly in time, but detrains water at its down-slope side through the friction layer. This two part structure is key to the dynamics of oceanic gravity currents. It is discussed in detail in Wirth (2009).

The first point we like to emphasise is the disastrous effect that the convective adjustment implemented in most ocean models has on the dynamics of gravity currents. When heavier water overlies lighter water a convective dynamics mixes the two water masses in a short time (see e.g. Wirth, 2009). In hydrostatic OGCMs this process is absent and a convective adjustment procedure is used that mixes the two water masses and their inertia. The convective adjustment used in our simulations does this by artificially augmenting the vertical diffusivity and viscosity to the value of $1 \mathrm{~m}^{2} \mathrm{~s}^{-1}$. Increasing only the diffusivity and leaving the viscosity unchanged is contrary to the fact that the turbulent Prandtl number is order unity. This procedure is found to mimic very well the convective dynamics at the ocean surface but has a disastrous effect on the dynamics of gravity currents. Indeed, at the downslope front of the gravity current the down-slope velocity decreases in the vicinity of the floor and heavy gravity current water superposes lighter ambient water, which triggers convective adjustment. The high vertical viscosity then inhibits a downslope movement of the gravity current and a vertical wall of dense water develops at the down-slope side of the gravity current as shown in Fig. 4, this is an artifact of the convective adjustment procedure. A comparison to Fig. 2 shows clearly the completely different dynamics due to the convective adjustment and demonstrates that it should not be used in gravity current calculations. The experiment involving convective adjustment will not be further discussed in the sequel.

The rate of descent of the gravity current is the most important property, its time evolution is given in Fig. 5. The rate of descent is defined by the movement of the x-component of the centre of gravity $c_{\mathrm{x}}$ of the gravity current. It is defined as:

$c_{\mathrm{x}}=\frac{\int \times\left(T-T_{0}\right) d A}{\int\left(T-T_{0}\right) d A}$,

where $T_{0}$ is the temperature of the surrounding water and the whole area, in the 2.5-D case, or the whole volume, in the 3-D case, is denoted by $A$.

A conspicuous feature is the strong resemblance between the experiments having at least three levels in the zone $\mathrm{Z} 1$ 


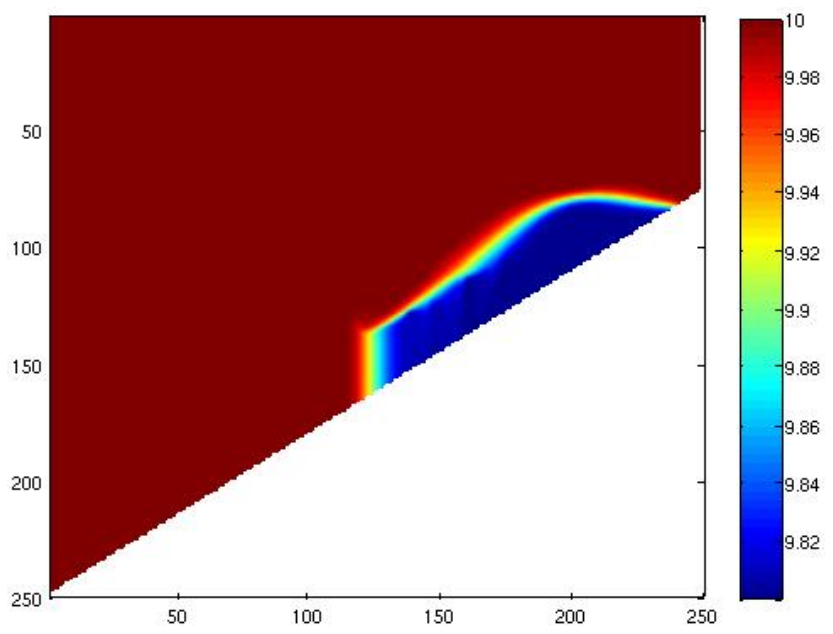

Fig. 4. Temperature structure (in $\mathrm{C}^{\circ}$ ) in the simulation with convective adjustment (G09). Coordinates give grid levels.

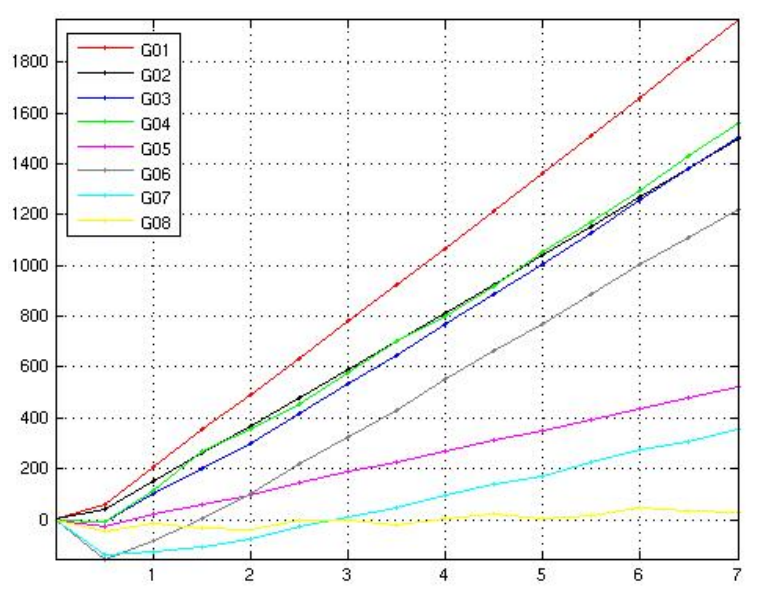

Fig. 5. Down-slope displacement of the centre of gravity of the gravity current in the 2.5-D experiments during the first 7 days of the experiments. Vertical axis in metres and horizontal axis in days.

(G01, G02, G03, G04, G06) showing a stronger descent than the experiments with a feeble resolution and proving the importance of the PBL dynamics (already emphasised in Wirth and Verron, 2008 and Wirth, 2010). It is striking that:

(i) the resolution in zones $\mathrm{Z} 2$ and $\mathrm{Z} 3$ have only a negligible influence, that

(ii) only three layers in the zone $\mathrm{Z1}$ are sufficient, and that

(iii) the horizontal resolution is not key (see experiment G04).

Simulations with only one level in the zone Z1 are clearly insufficient, they all lead to a descent rate that is smaller by at least a factor of two as compared to the reference calculation. the higher descent rate of the $\mathrm{z}$-coordinate experiment (G01) is due to the increased thickness of the friction

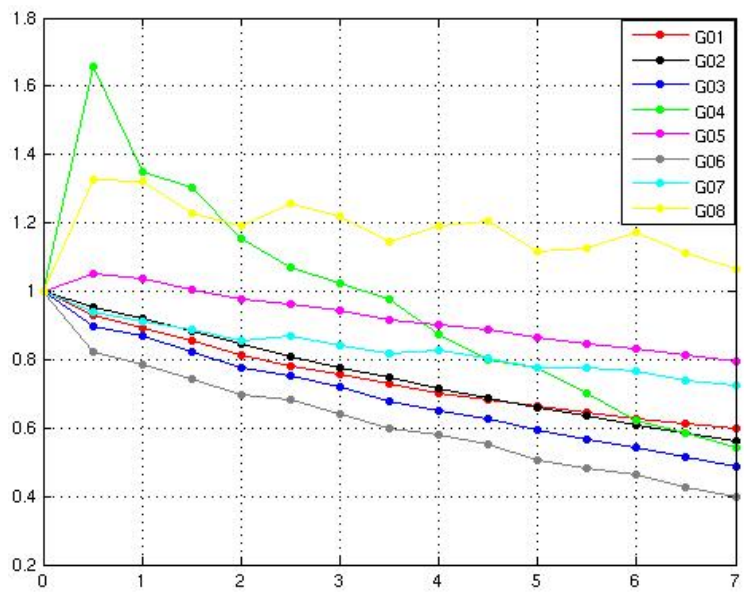

Fig. 6. Along-slope transport of the gravity current (normalised by the initial geostrophic value) in the 2.5 -D experiments during the first 7 days of the experiments.

layer, increased by spurious numerical diffusion along the horizontal direction (see Fig. 2).

We like to mention however, that it is not the rate of descent alone that is key but also the distribution of the descent is of paramount importance. In fact as we see in Fig. 2 most of the fluid descends in the friction layer at the down-slope side of the vein, whereas the bulk of the gravity current descends only slowly. This dynamics was explored in detail in Wirth (2009). This double-structure of the gravity current is key to the evolution of the density structure at the slope and can, of course, only be represented when the resolution at the topography is fine enough. Please see Sect. 6 for a discussion of the implications of the descent on the large scale circulation.

Another important parameter, although less important than the descent rate, is the along-slope transport of temperature. It is defined by:

$V_{T}=\int v\left(T-T_{0}\right) d A$.

Contrary to the downslope transport, which is performed in the PBL, the along-slope transport is done by the gravity current water above the vein, asking for a good resolution also in the zone $\mathrm{Z2}$. This increased resolution is provided in experiment G01, G02 and G03 and the good agreement of the along-slope transport in these experiments can be verified in Fig. 6.

When the Ekman layer is not resolved the veering (turning) of the velocity vectors in the vicinity of the wall is absent. The friction force exerted by the ocean floor is thus not only wrong in magnitude but also in direction. In the calculations with only 3 levels in the zone Z1 a correct Ekman veering is observed (not shown). 


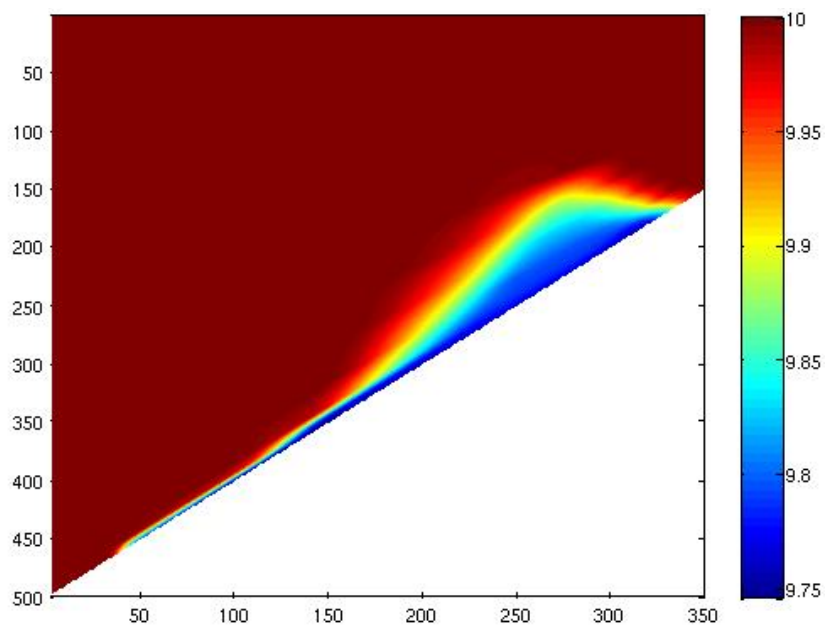

Fig. 7. Temperature structure (in $\mathrm{C}^{\circ}$ ) in simulation $(\mathrm{G} 11)$ after 7 days. Coordinates give grid levels.

\subsection{Results 3-D}

The dynamics in the 3-D case can clearly be divided in two phases: a laminar phase during the first 5 to 7 days followed by a dynamics dominated by strong eddies. The early behaviour somehow resembles the 2.5-D dynamics, but with the appearance of wave like disturbances on the gravity current that favour the downslope movement. When the temperature in the 3-D cases is averaged in the along slope direction the picture qualitatively resembles the 2.5-D results as can be seen by comparing Fig. 7 to Fig. 2 . The wave length of the instability is a little over $5 \mathrm{~km}$ in all experiments, which is very close to predicted value of $L=\sqrt{g^{\prime} h} / f$. As in the 2.5$\mathrm{D}$ experiments, the downslope dynamics in the first phase is strongly dependent on the resolution in the PBL, with an increased down-slope movement with a better resolution (see Fig. 8). The three dimensionality increases the downslope movement, in this early phase, by about $30 \%$ when compared to G01 and by $70 \%$ when compared to G11. The down-slope movement with a sufficient resolution (G11) of the PBL is found to be about 8-times larger than that of the case with a classical resolution (G13). The along slope movement in this early phase is very similar to the 2.5 -D experiments as can be verified in Fig. 9. An increased resolution leads to a smaller along slope transport. The dynamics similar to the corresponding 2.5-D experiments is followed, after a little more than 5 to 7 days (depending on the experiment), by a generation of strong eddies leading to a fully 3-D dynamics with an over three fold increase (not shown) of the downslope movement in the reference experiment (G11). The generation of eddies in gravity currents is a conspicuous feature and is explored in observations (Jungclaus et al., 2001), laboratory experiments (Whitehead et al., 1990) and numerical simulations (Legg et al., 2006). The cross over from one dynamics to the other is conspicuous in all the variables. In the

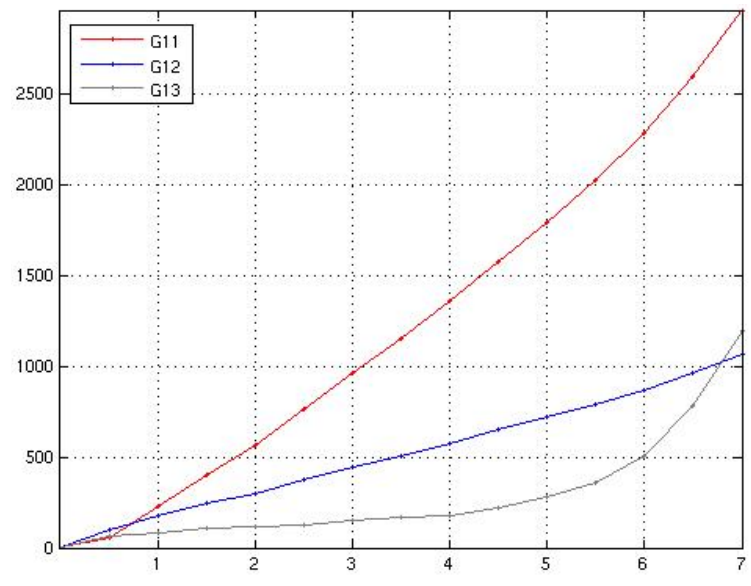

Fig. 8. Down-slope displacement of the centre of gravity of the gravity current in the 3-D experiments during the first 7 days of the experiments. Vertical axis in metres and horizontal axis in days.

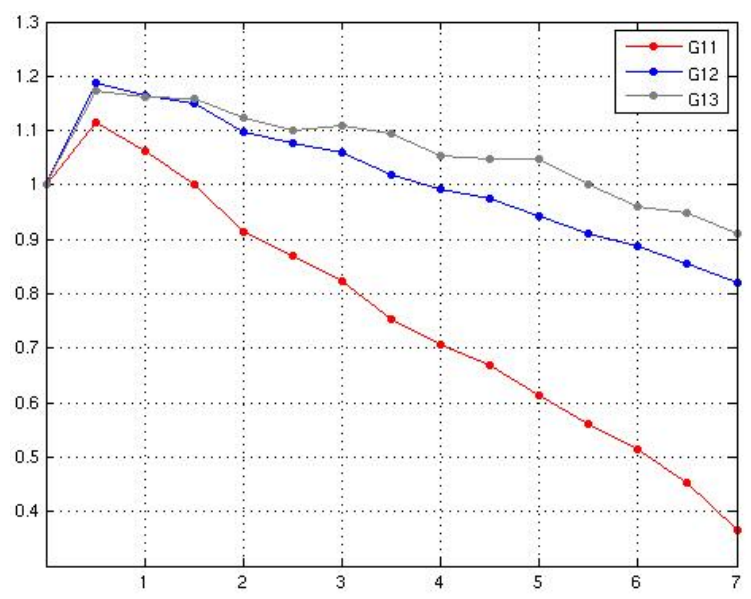

Fig. 9. Along-slope transport of the gravity current in the 3-D experiments during the first 7.5 days of the experiments.

experiments some of the gravity current water reached the boundary on the lower side of the domain after only a little more than five days and the dynamics of the downslope and along slope dynamics is altered. Furthermore, our resolution in the zone $\mathrm{Z} 3$ is too sparse in all experiments to allow for a detailed evaluation of the eddy dynamics. These two reasons prevent an analysis of the eddying regime.

\section{Discussion}

Our results demonstrate, that a better grid resolution in the PBL is key to the representation of the dynamics of oceanic gravity currents in numerical models. Our study shows, that only a few $(\approx 5)$ additional $\sigma$-levels are necessary to obtain a large improvement in the representation of the PBL dynamics. Such increase represents a raise of only $10 \%$ of 
calculation time in a typical state-of-the-art ocean model. The research presented here concentrates on the laminar dynamics of gravity currents and the development of its instability. The early phase, before the generation of large scale eddies after more than 5 days, is important as it represents the initial downslope movement and influences the subsequent generation of the eddies. The results are explained based on solid dynamical arguments (dynamics and resolution of the Ekman layer and the vein) and can be extrapolated to gravity currents with different slopes and density (temperature) anomalies. Furthermore, we see no reason why the here presented results for the dynamics of gravity currents can not be extrapolated to other processes near the ocean floor and to the interaction of ocean dynamics with topography in general.

The finding, that convective adjustment procedure destroys gravity current dynamics, blocking the downslope movement, is key to the representation of gravity currents and numerical simulations of ocean dynamics in high latitudes in general, which is strongly influenced by the descent of dense water.

Our results come as no surprise, the importance of the friction layer is already emphasised in Wirth (2009). It has a thickness a little larger than the Ekman layer thickness. If the numerical resolution does not allow for its resolution the dynamics of the gravity current can not be correctly represented. Most important, it is not the increase of the vertical viscosity, that enables the downslope movement of the gravity current, as put forward in recent publications, but the resolution of the Ekman layer dynamics. Such resolution can be obtained by increasing either the vertical viscosity or the vertical resolution near the ocean floor. The latter is the sensible way to go. It is not the physics that has to be adjusted to the numerics, but the numerics should respect the physics. We like to emphasise, that an increase of the vertical viscosity, leading to a thicker PBL (instead of increasing the vertical resolution, both leading to a better resolved PBL dynamics), is not a solution to the problem for still another reason. The Ekman transport in a laminar PBL is a function of the vertical viscosity $\left(\propto \sqrt{\nu_{v}}\right)$ and the dynamics of the gravity current above the PBL is governed by vertical Ekman pumping due to the divergence in the Ekman transport. Artificially increasing the vertical viscosity is clearly the wrong thing to do. Please note that the situation is very different to the increase of the horizontal viscosity to allow for a resolution of the horizontal boundary layer, the Munk-layer. The transport in the Munk layer is, however, imposed by the interior Sverdrup dynamics and does, to leading order, not depend on the horizontal viscosity.

It is not only the rate of descent of the centre of gravity that is important. The descent in a fine layer changes the density all along the slope but less massively than a descent of the gravity current as a bulk, the latter leading to a larger density anomaly but more locally. It is the density structure at the boundary that determines the baroclinic transport in geostrophic theory. The density structure is in large areas of the world's oceans determined by gravity current dynamics. Examples are gravity currents all along the coast of Antarctica. Getting the gravity current dynamics wrong means getting the geostrophic large scale dynamics wrong, that means getting it wrong to leading order.

Acknowledgements. Comments from and discussions with Patrick Marchesiello, Guervan Madec, Joel Sommeria, Julien Le Sommer and Jan Zika were key in writing this paper. This work is part of the COUGAR project funded by ANR-06-JCJC-0031-01 and the European Integrated Project MERSEA by the Région Rhône-Alpes (GRANT CPER0713 CIRA: http://www.ci-ra.org/).

Edited by: J. M. Huthnance

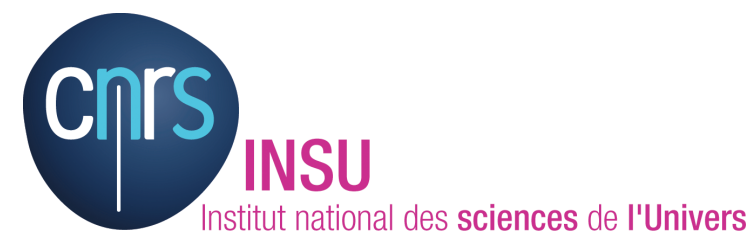

The publication of this article is financed by CNRS-INSU.

\section{References}

Coleman, G. N., Ferziger, J. H., and Spalart, P. R.: A numerical study of the turbulent Ekman layer, J. Fluid. Mech., 213, 313348, 1990.

Ezer, T.: Entrainment, diapycnal mixing and transport in threedimensional bottom gravity current simulations using the MellorYamada turbulence scheme, Ocean Model., 9, 151-168, 2005.

Ezer, T. and Mellor, G. L.: A generalized coordinate ocean model and a comparison of the bottom boundary layer dynamics in terrain-following and in z-level grids, Ocean Model., 6, 379-403, 2004.

Ezer, T. and Weatherly, G. L.: A numerical study of the interaction between a deep cold jet and the bottom boundary layer of the ocean? J. Phys. Oceanogr., 20, 801-816, 1990.

Griffiths, R. W.: Gravity currents in rotating systems, Ann. Rev. Fluid Mech., 18, 59-89, 1986.

Jungclaus, J. H.: A three-dimensional simulation of the formation of anticyclonic lenses (meddies) by the instability of an intermediate depth boundary current, J. Phys. Oceanogr., 29, 1579-1598, 1999.

Jungclaus, J. H., Hauser, J., and Käse, R. H.: Cyclogenesis in the Denmark Strait overflow plume, J. Phys. Oceanogr., 31, 32143229, 2001.

Killworth, P. D. and Edwards, N. R.: A turbulent bottom boundary layer code for use in numerical ocean models, J. Phys. Oceanogr., 29, 1221-1238, 1999.

Legg, S., Hallberg, R. W. and Girton, J. B.: Comparison of entrainment in overflows simulated by z-coordinate, isopycnal and non-hydrostatic models, Ocean Model., 11, 69-97, 2006.

Legg, S., Jackson, L., and Hallberg, R. W.: Eddy resolving modeling of overflows, Geophys. Monog. Series, 117, 63-81, 2008. 
Madec, G.: Nemo ocean Engine, Institut Pierre Simon Laplace (IPSL), France, http://www.nemo-ocean.eu/About-NEMO/ Reference-manuals, last access: June 2010, No 27, ISSN No 1288-1619, 2008.

Pedlosky, J.: Ocean Circulation Theory, Springer, 453 ps. ISBN: 3540-60489-8, 1998.

Whitehead, J. A., Stern, M. E., Flierl, G. R., and Klinger, B. A.: Experimental observations of baroclinic eddies on a sloping bottom, J. Geophy. Res., 95, 9585-9610, 1990.

Willebrand, J., Barnier, B., Böning, C., Dieterich, C., Killworth, P., Le Provost, C., Jia, Y., Molines, J. M., and New, A. L.: Circulation characteristics in three eddy-permitting models of the North Atlantic, J. Prog. Oceanogr., 48, 123-162, 2001.

Winton, M., Hallberg, R., and Gnanadesikan, A.: Simulation of density-driven frictional downslope flow in z-coordinate ocean modelset, J. Phys. Oceanogr., 28, 2163-2174, 1998.
Wirth, A.: A non-hydrostatic flat-bottom ocean model entirely based on Fourier expansion, Ocean Model., 9, 71-87, 2004.

Wirth, A.: On the basic structure of oceanic gravity currents, Ocean Dynam., 59, 551-563, doi:10.1007/s10236-009-0202-9, 2009.

Wirth, A.: Estimation of Friction Parameters in Gravity Currents by Data Assimilation in a Model Hierarchy, submitted to Ocean Dynam., 2010.

Wirth, A. and Verron, J.: Estimation of Friction Parameters and Laws in 1.5D Shallow-Water Gravity Currents on the fPlane, by Data Assimilation Ocean Dynam., 58, 247-257, doi:10.1007/s10236-008-0151-8, 2008.

Wirth, A. and Sommeria, J.: Gravity current experiments on the Coriolis platform, Technical report EPSHOM, 2007. 